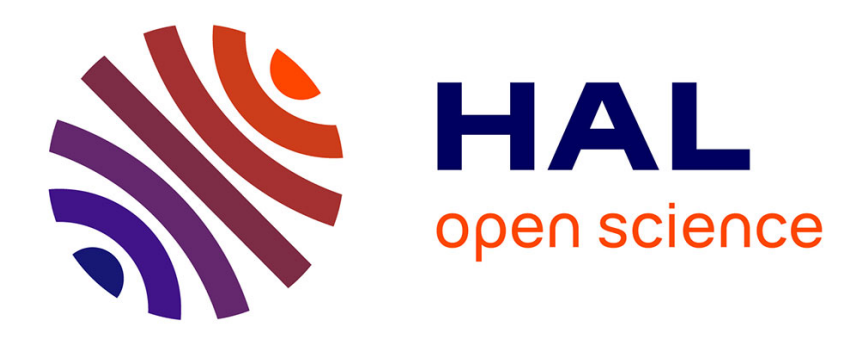

\title{
Reversing the drift of the Ehrenfest urn model and three conditionings
}

Thierry Huillet

\section{To cite this version:}

Thierry Huillet. Reversing the drift of the Ehrenfest urn model and three conditionings. Journal of Physics A: Mathematical and Theoretical, 2009, 42 (34), pp.345005. hal-00392311

\section{HAL Id: hal-00392311 \\ https://hal.science/hal-00392311}

Submitted on 7 Jun 2009

HAL is a multi-disciplinary open access archive for the deposit and dissemination of scientific research documents, whether they are published or not. The documents may come from teaching and research institutions in France or abroad, or from public or private research centers.
L'archive ouverte pluridisciplinaire HAL, est destinée au dépôt et à la diffusion de documents scientifiques de niveau recherche, publiés ou non, émanant des établissements d'enseignement et de recherche français ou étrangers, des laboratoires publics ou privés. 


\title{
Reversing the drift of the Ehrenfest urn model and three conditionings
}

\author{
Thierry Huillet \\ Laboratoire de Physique Théorique et Modélisation, \\ CNRS-UMR 8089 et Université de Cergy-Pontoise, \\ 2 Avenue Adolphe Chauvin, F-95302, Cergy-Pontoise, France \\ Thierry.Huillet@u-cergy.fr
}

\begin{abstract}
We consider a drift-reversed version of the celebrated Ehrenfest urn process with $N$ balls. For this 'dual' process, the boundaries are assumed to be absorbing and so the killing times at the boundaries play a central role. Three natural conditionings on the fixation/extinction events pertaining to this model are investigated. Some spectral informations on the conditioned Markoff chains are obtained, allowing to draw precise new conclusions on their limiting behaviors.
\end{abstract}

KEYWORDS: Ehrenfest urn model, drift reversal, conditioning, fluctuation theory, fixation/extinction times.

\section{Introduction and outline of the results.}

The Ehrenfest urn model (alias 'dogs and fleas') is a famous physical ball-in-box model for the heat exchange between two bodies (Urns) at unequal temperature (the number of balls labeled 1 to $N$ within each Urn). See [6]. It was designed to elucidate some paradoxes raised by Boltzmann while considering the kinetic theory of gases, among which irreversibility versus recurrence of states. See [17] for a review. The relevant state $X_{n}$ counts the number of balls within say Urn 1 after $n$ steps, one interchanging step consisting in selecting at random a number within $\{1, . ., N\}$, determining the Urn where the ball with this number is and moving this ball to the other Urn. As is well-known, $X_{n}$ is a standard positive-recurrent, 2-periodic birthand-death Markoff chain with state-space $\{0, . ., N\}$. This simple model is an exactly solvable Markoff chain because its spectral representation was completely determined after Kac and Krawtchouk, among others. For instance, the composition of Urn 1 probability at each time is exactly known. See [12], [7], [14] and [9]. The mathematician Mark Kac wrote about this model that it was: “...probably one of the more instructive models in the whole of Physics...", [13].

In this Note, we shall rather be concerned in a related ball-in-box model which is 'dual' to that of Ehrenfest in the sense that the chain local probabilities to move up and down are being switched in the bulk of the state-space, which amounts to reversing the drift of $X_{n}$. See [4] where this type of duality was coined the 'Wall duality', although it concerns there birth and death chains evolving on a semi-infinite state-space with a reflecting barrier. For our drift-reversed model, the boundaries $\partial:=\{0, N\}$ will be assumed to be absorbing. In terms of stochastics, 
this problem may be recast as a new birth-and-death Markoff chain, say $\widehat{X}_{n}$, still with state-space $\{0, . ., N\}$ but which is being absorbed at $\partial$. We shall therefore let $\widehat{\tau}_{x}:=\inf \left(n: \widehat{X}_{n} \in \partial\right.$ given $\left.\widehat{X}_{0}=x\right)$ be its first hitting time of $\partial$ when started at $x$. This model was first considered in [10] from a purely combinatorial point of view and was coined the 'Mabinogion urn model' for reasons explained therein. The physical motivations were to design a simple model of the spread of influences amongst versatile populations and draw some conclusions about the hitting times of the boundaries for various initial conditions of interest, together with some limit laws as $N$ gets large. We wish here to shed some new light on this dual process that rather focuses on conditioning $\widehat{X}_{n}$ on the fixation/extinction events at the boundaries. We refer to [2], [11] for similar concerns in population genetics.

We shall therefore investigate the three following conditional problems pertaining to $\widehat{X}_{n}$ and of general interest:

(i) Consider the drift-reversed urn process $\widehat{X}_{n}$, started at $\widehat{X}_{0}=x \in\{1, . ., N-1\}$. Recall $\widehat{\tau}_{x}$ is its absolute extinction time at $\partial$. Then, conditional on the event $\left(\widehat{\tau}_{x}>n\right)$, $\widehat{X}_{n}$ turns out to be a positive recurrent Markov chain on $\{1, \ldots, N-1\}$ whose Yaglom invariant (stationary) measure will be shown to be uniform. This measure is also the quasi-stationary distribution of the chain.

(ii) A more stringent conditioning is also investigated: assuming the chain $\widehat{X}_{n}$ given $\widehat{X}_{0}=x$, to be conditioned on never getting killed in the distant future, this conditional process is also a positive recurrent Markov chain on $\{1, \ldots, N-1\}$ but whose invariant measure will now be shown to be binomial $\operatorname{bin}(N-2,1 / 2)$. Curiously, its probability transition matrix turns out to be the one of a reversible Ehrenfest urn process but with $N-2$ balls.

Both results make use of a simple duality result displayed in Lemma 1. This property makes the invariant laws of the conditioned processes explicit which is a rare event in the context of quasi-stationary distributions for finite birth and death chains, [16].

(iii) Finally, we shall consider the drift-reversed random walk $\widehat{X}_{n}$ conditioned on exit through state $\{N\}$ using an appropriate Doob-transform built on the harmonic (or scale) function of the chain. We shall use this transformation to give some information on the time it takes for this conditioned random walk (RW) to undergo a sweep, that is to move from one endpoint of the state-space to the other opposite absorbing state. Our results constitute a drift-reversed refined version of a result in [1] for the usual Ehrenfest model.

\section{Preliminary: Drift reversal of the Ehrenfest urn model.}

The Ehrenfest urn model is a birth and death (BD) process on $\{0, \ldots, N\}$, say $X_{n}$, whose up and down local transition probabilities $X_{n} \rightarrow X_{n} \pm 1$ given $X_{n}=x$ are given by: $p_{x}=1-x / N, x=0, \ldots, N-1, q_{x}=x / N, x=1, \ldots, N$. Its one-step transition matrix therefore is of the Jacobi type (empty entries are 0)

$$
P=\frac{1}{N}\left[\begin{array}{ccccccc}
0 & N & & & & & \\
1 & 0 & N-1 & & & \\
2 & 0 & N-2 & & \\
& \ddots & \ddots & \ddots & \\
& & N-1 & 0 & 1 \\
& & & N & 0
\end{array}\right] .
$$


The set of the eigenvalues of $P$ has been known for long to be $\lambda_{k}=1-\frac{2 k}{N}, k=0, . ., N$. See [12] for example. The Ehrenfest urn process is well-known to be positive-recurrent and 2-periodic with symmetric binomial $\operatorname{bin}(N, 1 / 2)$ invariant measure.

By drift reversal (Wall duality), let us exchange the $p_{x}$ and $q_{x}$ within the statespace bulk $\{1, \ldots, N-1\}$ and force the states 0 and $N$ to be absorbing. The reversed chain, say $\widehat{X}_{n}$, is a new BD chain with transition probabilities $\widehat{p}_{x}=q_{x}, \widehat{q}_{x}=p_{x}$, $x=1, \ldots, N-1$. We obtain the stochastic transition matrix of the Mabinogion urn model with dimensions $(N+1) \times(N+1)$ :

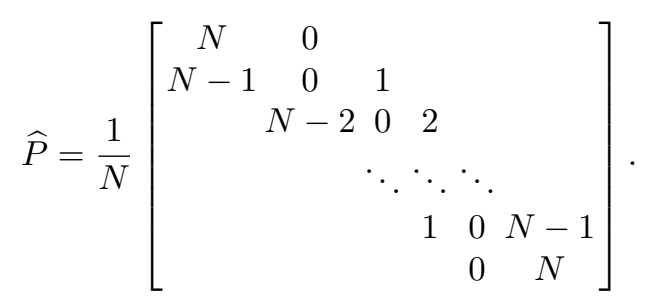

The drift of the random walk $\widehat{X}_{n}$ is thus

$$
\mathbb{E}_{\widehat{X}_{n}=x}\left(\widehat{X}_{n+1}-\widehat{X}_{n}\right)=\widehat{p}_{x}-\widehat{q}_{x}=-\left(p_{x}-q_{x}\right)=-\mathbb{E}_{X_{n}=x}\left(X_{n+1}-X_{n}\right)
$$

but the conditional local fluctuations

$$
\sigma_{\widehat{X}_{n}=x}^{2}\left(\widehat{X}_{n+1}-\widehat{X}_{n}\right)=4 \widehat{p}_{x} \widehat{q}_{x}=4 p_{x} q_{x}=\sigma_{X_{n}=x}^{2}\left(X_{n+1}-X_{n}\right)
$$

are left unchanged.

Let us delete the first line and column of $\widehat{P}$. We obtain the matrix of reduced size $(N-1) \times(N-1)$

$$
\widetilde{P}=\frac{1}{N}\left[\begin{array}{ccccccc}
0 & 1 & & & & \\
N-2 & 0 & 2 & & & \\
& N-3 & 0 & 3 & & \\
& & & \ddots & \ddots & \ddots & \\
& & & & 2 & 0 & N-2 \\
& & & & 1 & 0
\end{array}\right]
$$

Let $1:=(1,1, . ., 1)^{\prime}$ be a column vector of $1 \mathrm{~s}$ with ' standing for transposition. The matrix $\widetilde{P}$ is substochastic with

$$
\widetilde{P} \mathbf{1}=\frac{1}{N}(1, N, N, \ldots, N, 1)^{\prime}
$$

therefore it looses mass through the states $\{1\}$ and $\{N-1\}$. Let us now emphasize the following obvious but remarkable fact:

Lemma 1 With $\widetilde{P}^{\prime}$ the transpose of $\widetilde{P}$, it holds that

$$
\frac{N}{N-2} \widetilde{P}^{\prime}=\frac{1}{N-2}\left[\begin{array}{cccccc}
0 & N-2 & & & \\
1 & 0 & N-3 & & \\
& 2 & 0 & N-4 & \\
& & \ddots & \ddots & \ddots & \\
& & & N-3 & 0 & 1 \\
& & & & N-2 & 0
\end{array}\right]
$$

so that $\frac{N}{N-2} \widetilde{P}^{\prime}$ is again an Ehrenfest transition matrix but with $N-2$ balls. 
From this result, we may draw the following spectral conclusions that will be used in the sequel: The spectrum of $\frac{N}{N-2} \widetilde{P}^{\prime}$ is $1-2(k-1) /(N-2), k=1, . ., N-1$. The one of $\widetilde{P}^{\prime}$ and therefore of $\widetilde{P}$ is

$$
\lambda_{k}=\frac{N-2}{N}\left(1-2 \frac{k-1}{N-2}\right)=1-\frac{2 k}{N}, k=1, . ., N-1 .
$$

The spectrum of $\widehat{P}$ is $1-\frac{2 k}{N}, k=1, . ., N-1$ to which one should add the pair $\{1,1\}$ corresponding to the two absorbing states $\{0, N\}$. The dominant eigenvalue of $\widehat{P}$ just after the double eigenvalue $\{1\}$ therefore is $1-2 / N$.

\section{Drift reversal and quasi-stationary distribution.}

Let $\widehat{P}$ be the $(N+1) \times(N+1)$ forward Jacobi transition matrix of the Mabinogion urn process. For such a discrete Markov chain, say $\widehat{X}_{n}$, started at $\widehat{X}_{0}=x$, the hitting time $\widehat{\tau}_{x}$ of the absorbing boundaries $\{0, N\}$ is finite with probability 1 . We have $\widehat{\tau}_{x}=\widehat{\tau}_{x, 0} \wedge \widehat{\tau}_{x, N}$, the infimum of the hitting times of states $\{0\}$ and $\{N\}$, starting from $x$.

Let $\widetilde{P}$ be the $(N-1) \times(N-1)$ sub-matrix of $\widehat{P}$ obtained by removing the states $\{0, N\}$ from the entries of $\widehat{P}$. It is sub-stochastic at states $\{1, N-1\}$ with entries satisfying (1). The matrix $\widetilde{P}$ is easily seen to be the transition matrix of the process $\widehat{X}_{n} \cdot \mathbf{1}\left(\widehat{\tau}_{x}>n\right)$ (that is $\widehat{X}_{n}$, restricted to the set $\left.\widehat{\tau}_{x}>n\right)$. In other words,

$$
\widetilde{P}^{n}(x, y)=\mathbb{P}_{x}\left(\widehat{X}_{n}=y ; \widehat{\tau}_{x}>n\right) .
$$

Let $\rho<1$ be the spectral radius of $\widetilde{P}$, with from Lemma 1: $\rho=1-2 / N$. The matrix $\widetilde{P}$ is irreducible with period 2 whereas $\widetilde{P}^{2}$ is irreducible and aperiodic, with spectral radius $\rho^{2}$. By Perron-Frobenius theorem for primitive non-negative matrices

$$
\lim _{n \uparrow \infty} \rho^{-2 n} \widetilde{P}^{2 n}=\boldsymbol{\psi} \boldsymbol{\mu}^{\prime},
$$

where $\boldsymbol{\mu}^{\prime}>\mathbf{0}(\boldsymbol{\psi}>\mathbf{0})$ is the left- (right- ) line (column) eigenvector of $\widetilde{P}$ associated to $\rho$, defined by: $\boldsymbol{\mu}^{\prime} \widetilde{P}=\rho \boldsymbol{\mu}^{\prime}$ and $\widetilde{P} \boldsymbol{\psi}=\rho \boldsymbol{\psi}$. Let us fix the normalization of the Perron vectors as follows: We will choose $\boldsymbol{\mu}$ so that it constitutes a probability distribution vector so with: $\sum_{x=1}^{N-1} \mu(x)=1$. We will also choose $\boldsymbol{\psi}$ so that $\boldsymbol{\mu}^{\prime} \boldsymbol{\psi}=1$. It results from $(2,3)$ that

$$
\lim _{n \uparrow \infty} \rho^{-2 n} \mathbb{P}_{x}\left(\widehat{\tau}_{x}>2 n\right)=\psi(x)
$$

so that $\widehat{\tau}_{x}$ has geometric tails with exponent $\rho$ : on the set $\left(\widehat{\tau}_{x}>2 n\right)$, the Markov chain $\widehat{X}_{2 n}$ with values in $\{1, . ., N-1\}$ is called geometrically transient. The latter formula gives the limiting interpretation of $\boldsymbol{\psi}$ in our context. We also note from $(2$, 3) that,

$$
\mathbb{P}_{\boldsymbol{\mu}}(\widehat{\tau}>2 n):=\sum_{x=1}^{N-1} \mu(x) \mathbb{P}_{x}\left(\widehat{\tau}_{x}>2 n\right)=\rho^{2 n} .
$$

If the process is started with $\boldsymbol{\mu}$, the law of the hitting time $\widehat{\tau}$ of $\partial$ is exactly geometric $(\rho)$ distributed on $\{2,4, .$.$\} .$

Consider now the conditional probability $\mathbb{P}_{x}\left(\widehat{X}_{2 n}=y \mid \widehat{\tau}_{x}>2 n\right)$. One easily can check from $(2,3,4)$ that, independently of the starting point $x$

$$
\mathbb{P}_{x}\left(\widehat{X}_{2 n}=y \mid \widehat{\tau}_{x}>2 n\right) \underset{n \uparrow \infty}{\rightarrow} \mu(y)
$$


As such, the probability measure $\boldsymbol{\mu}$ interprets as a Yaglom limit of $\widehat{X}_{n}$. See [18]. Furthermore, for each $n$

$$
\mathbb{P}_{\boldsymbol{\mu}}\left(\widehat{X}_{2 n}=y \mid \widehat{\tau}>n\right)=\mu(y), y \in\{1,2, . ., N-1\}
$$

and $\boldsymbol{\mu}$ is the (unique) quasi-stationary distribution of $\widehat{X}_{n}$ : As is well-known for Markov chains with finite state-space, the Yaglom limit coincides with the quasistationary distribution (qsd). See [16] and [3] for qsd examples in much more general contexts.

Applying these general ideas to the Mabinogion urn model, one obtains:

Theorem 2 (i) For the Mabinogion urn model, the Yaglom limit of the process conditioned on not yet being killed at the current time is the uniform distribution on the set $\{1, . ., N-1\}$ :

$$
\boldsymbol{\mu}^{\prime}=(1 /(N-1), . ., 1 /(N-1)) .
$$

(ii) With $\widehat{\tau}_{x}=\widehat{\tau}_{x, 0} \wedge \widehat{\tau}_{x, N}$, for each $x \in\{1, . ., N-1\}$, we have

$$
\lim _{n \uparrow \infty}(1-2 / N)^{-2 n} \mathbb{P}_{x}\left(\widehat{\tau}_{x}>2 n\right)=(N-1) \cdot 2^{-(N-2)}\left(\begin{array}{c}
N-2 \\
x-1
\end{array}\right),
$$

and, with $\boldsymbol{\mu}$ uniform on $\{1, . ., N-1\}$ :

$$
\mathbb{P}_{\boldsymbol{\mu}}(\widehat{\tau}>2 n)=(1-2 / N)^{2 n} .
$$

Proof: In our case study, $\rho=1-2 / N$ (the third largest eigenvalue after $\lambda_{0}=$ $\lambda_{1}=1$ for $\widehat{P}$ which is also the dominant eigenvalue of $\left.\widetilde{P}\right)$ and $\boldsymbol{\mu}=1 /(N-1) \cdot \mathbf{1}$ can be checked to satisfy: $\boldsymbol{\mu}^{\prime} \widetilde{P}=\left(1-\frac{2}{N}\right) \boldsymbol{\mu}^{\prime}$ and so the Yaglom limit is the uniform distribution. Finally, the right-eigenvector $\psi$ associated to $\widetilde{P}$ and $\rho=1-2 / N$ can easily be checked to be: $\psi(x)=K \cdot\left(\begin{array}{c}N-2 \\ x-1\end{array}\right), x=1, . ., N-1$, for some constant $K=(N-1) \cdot 2^{-(N-2)}$ consistently with $\boldsymbol{\mu}^{\prime} \boldsymbol{\psi}=1$.

\section{Conditioning on no-exit in the remote future.}

Consider now the proper Markov chain whose transition probability matrix $\widetilde{\Pi}$ is obtained from $\widetilde{P}$ by the Doob transform:

$$
\widetilde{\Pi}(x, y)=\rho^{-1} \frac{\psi(y)}{\psi(x)} \widetilde{P}(x, y), x, y \in\{1, . ., N-1\},
$$

satisfying $\widetilde{\Pi} \mathbf{1}=\mathbf{1}$. In matrix form, this transformation reads: $\widetilde{\Pi}=\rho^{-1} D_{\psi}^{-1} \widetilde{P} D_{\psi}$.

The invariant probability distribution $\widetilde{\boldsymbol{\pi}}$ on $\{1, . ., N-1\}$ satisfying: $\widetilde{\boldsymbol{\pi}}^{\prime} \widetilde{\Pi}=\widetilde{\boldsymbol{\pi}}^{\prime}$ exists. Clearly, it is given explicitly by:

$$
\widetilde{\pi}(x)=\psi(x) \mu(x), x=1, . ., N-1
$$

where $\boldsymbol{\mu}$ and $\boldsymbol{\psi}$ are defined in (3). The Markov chain with transformed transition probability matrix $\widetilde{\Pi}$ is the one of the process whose $n$-step probability distribution is:

$$
\lim _{n^{\prime} \uparrow \infty} \mathbb{P}_{x}\left(\widehat{X}_{n}=y \mid \widehat{\tau}_{x}>n^{\prime}\right) .
$$

It corresponds to $\widehat{X}_{n}$ conditioned to never hit the coffin state $\partial$ in the distant future. This process has a unique invariant measure given by $\widetilde{\boldsymbol{\pi}}$ in (9). This second conditioning being more stringent than the previous one, one should intuitively charge more heavily the sample paths staying away from $\{0, N\}$. We indeed have: 
Theorem 3 (i) For the Mabinogion urn model, the invariant measure of the corresponding process conditioned on never getting killed in the remote future is binomial $\operatorname{bin}(N-2,1 / 2)$, namely:

$$
\widetilde{\pi}(x)=2^{-(N-2)}\left(\begin{array}{c}
N-2 \\
x-1
\end{array}\right), x=1, . ., N-1 .
$$

(ii) For the Mabinogion urn model with $N$ balls, the transition matrix of the corresponding conditioned process is the one of the Ehrenfest urn model with $N-2$ balls.

Proof: (i) The limiting qsd $\boldsymbol{\mu}$ was shown to be uniform and the right-eigenvector $\psi$ associated to $\widetilde{P}$ and $\rho=1-2 / N$ was checked to be: $\psi(x)=K \cdot\left(\begin{array}{c}N-2 \\ x-1\end{array}\right), x=1, . ., N-$ 1 where $K=(N-1) \cdot 2^{-(N-2)}$. (11) follows from (9). Note that, in contrast with the uniform Yaglom limit law $\boldsymbol{\mu}$, the probability mass of $\widetilde{\pi}$ is more concentrated on the central atoms close to $N / 2$, away from $\{0, N\}$. (ii) follows from $\widetilde{\Pi}=\rho^{-1} D_{\psi}^{-1} \widetilde{P} D_{\psi}$ and the expressions of $\boldsymbol{\psi}, \widetilde{P}$ and $\rho$. For instance, with $\widetilde{P}(x, x+1)=x / N$,

$$
\widetilde{\Pi}(x, x+1)=\frac{N}{N-2}\left(\begin{array}{c}
N-2 \\
x-1
\end{array}\right)^{-1} \frac{x}{N}\left(\begin{array}{c}
N-2 \\
x
\end{array}\right)=1-\frac{x}{N-2} .
$$

\section{Conditioning on exit in state $\{N\}$.}

Consider again the reversed BD chain, $\widehat{X}_{n}$ with transition matrix $\widehat{P}$ where both states $\{0, N\}$ are absorbing. Define the scale (or harmonic) function $\varphi$ of this BD random walk as the function which makes $M_{n}:=\varphi\left(\widehat{X}_{n \wedge \tau_{x, 0}}\right)$ a martingale. The function $\varphi$ is important because, as is well-known, for all $0<x<N$, with $\widehat{\tau}_{x}=\widehat{\tau}_{x, 0} \wedge \widehat{\tau}_{x, N}$ the first hitting time of $\{0, N\}$ starting from $x$

$$
\mathbb{P}_{x}\left(\widehat{X}_{\widehat{\tau}_{x}}=N\right)=\mathbb{P}\left(\widehat{\tau}_{x, N}<\widehat{\tau}_{x, 0}\right)=\frac{\varphi(x)}{\varphi(N)}
$$

gives a closed-form expression of the probability of exit at $\{N\}$ before hitting $\{0\}$. The searched harmonic function is $\varphi(x)=: 1+\sum_{y=1}^{x-1} \psi(y)$ where $\psi(y)$ satisfies: $\widehat{q}_{y} \psi(y-1)=\widehat{p}_{y} \psi(y)$, with $\psi(1):=1$. Thus $\psi(y)=\prod_{z=1}^{y} \frac{\widehat{q}_{z}}{\widehat{p}_{z}}$ and so, with $x=$ $1, \ldots, N, \varphi(0):=0$ :

$$
\varphi(x)=1+\sum_{y=1}^{x-1} \prod_{z=1}^{y} \frac{\widehat{q}_{z}}{\widehat{p}_{z}}=1+\sum_{y=1}^{x-1} \prod_{z=1}^{y} \frac{p_{z}}{q_{z}}=1+\sum_{y=1}^{x-1}\left(\begin{array}{c}
N-1 \\
y
\end{array}\right)
$$

is a cumulative binomial sequence. Note $\varphi(1)=1$. Let us consider the problem of conditioning $\widehat{X}_{n}$ on exiting in state $\{N\}$. Let $D_{\varphi}:=\operatorname{diag}(\varphi(0), \ldots, \varphi(N))$. The transition matrix $\widehat{Q}_{c}$ of this conditioned RW is given in terms of the Doob-transform, [5]:

$$
\widehat{Q}_{c}=D_{\varphi}^{-1} \widehat{P} D_{\varphi}
$$

As a result, the exact shape of $\widehat{Q}_{c}$ is

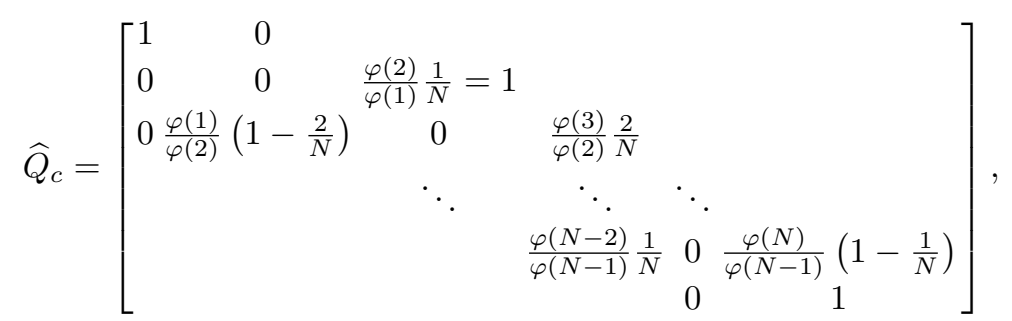


where it is apparent that state $\{0\}$ became isolated and disconnected in the transformation process $\left(\widehat{Q}_{c}(0,0)=1\right.$ and $\left.\widehat{Q}_{c}(0,1)=\widehat{Q}_{c}(1,0)=0\right)$. Deleting the line and the row $\{0\}$ of $\widehat{Q}_{c}$, we get a stochastic matrix, call it $\widehat{P}_{c}$, of a process say $\widehat{X}_{n}^{c}$ now on the state-space $\{1, \ldots, N\}$ which corresponds to $\widehat{X}_{n}$ conditioned to first hit state $\{N\}$ before state $\{0\}$. As required if state $\{0\}$ is to be inaccessible after conditioning, the state $\{1\}$ of this conditioned BD process now is purely reflecting $\left(\widehat{Q}_{c}(1,2)=1\right)$, whereas state $\{N\}$ remains absorbing $\left(\widehat{Q}_{c}(N, N)=1\right)$. $\widehat{Q}_{c}$ and $\widehat{P}$ are diagonally similar and so both share the same spectrum which is $\lambda_{k}=1-\frac{2 k}{N}, k=1, . ., N-1$ to which one should add the eigenvalues $\{1,1\}$. Putting this together, we get

Proposition 4 (i) The transition matrix of the Mabinogion urn model conditioned on exit at $\{N\}$ is the restriction $\widehat{P}_{c}$ of $\widehat{Q}_{c}$ to $\{1, \ldots, N\}$ where the scale function $\varphi$ is given by (13).

(ii) The spectrum of $\widehat{P}_{c}$ is reduced to: $\{1\} \cup\left\{1-\frac{2 k}{N}, k=1, . ., N-1\right\}$.

For this conditioned process, it is of importance to evaluate the time $\widehat{\tau}_{1, N}^{c}$ it takes to reach $\{N\}$ starting from state $\{1\}$, joining the two extreme states of this process. We have:

Theorem 5 (i) The probability generating function of $\widehat{\tau}_{1, N}^{c} \geq N-1$ is

$$
\mathbb{E}\left(z^{\widehat{\tau}_{1, N}^{c}}\right)=\prod_{k=1}^{N-1} \frac{\left(1-\lambda_{k}\right) z}{1-\lambda_{k} z}, z \in[0,1]
$$

where $-1<\lambda_{k}=1-\frac{2 k}{N}<+1, k=1, \ldots, N-1$ are the $N-1$ distinct eigenvalues of $\widehat{P}_{c}$, avoiding $\lambda_{0}=1$.

(ii) In particular,

$$
\mathbb{E}\left(\widehat{\tau}_{1, N}^{c}\right) \sim_{N \uparrow \infty} \frac{N}{2} \log N \text { and } \sigma^{2}\left(\widehat{\tau}_{1, N}^{c}\right) \sim_{N \uparrow \infty}\left(\frac{N}{2}\right)^{2} .
$$

(iii)

$$
\frac{\widehat{\tau}_{1, N}^{c}}{\mathbb{E}\left(\widehat{\tau}_{1, N}^{c}\right)} \underset{N \uparrow \infty}{\longrightarrow} 1 \text { in probability }
$$

Proof: $(i)$ is a particular incarnation of a result described in [15], [8] in the context of BD chains absorbed at $\{N\}$. This part makes use of our findings on the spectrum of $\widehat{P}_{c}$ which makes (15) explicit.

(ii) From $(i)$, we have $\mathbb{E}\left(\widehat{\tau}_{1, N}^{c}\right)=\sum_{k=1}^{N-1} \frac{1}{1-\lambda_{k}}$ and

$$
\sigma^{2}\left(\widehat{\tau}_{1, N}^{c}\right)=\sum_{k=1}^{N-1}\left(1-\lambda_{k}\right)^{-2}-\sum_{k=1}^{N-1}\left(1-\lambda_{k}\right)^{-1} .
$$

Because the eigenvalues $\lambda_{k}$ are known leading to: $1-\lambda_{k}=\frac{2 k}{N}$, using the integral approximation

we easily get

$$
\mathbb{E}\left(\widehat{\tau}_{1, N}^{c}\right) \sim N \int_{0}^{1} \frac{1}{2} \frac{d x}{(x+1 / N)},
$$

$$
\mathbb{E}\left(\widehat{\tau}_{1, N}^{c}\right) \sim \frac{N}{2} \log N \text { and } \sigma^{2}\left(\widehat{\tau}_{1, N}^{c}\right) \sim\left(\frac{N}{2}\right)^{2},
$$

showing that $\sigma^{2}\left(\widetilde{\tau}_{0, N} / \mathbb{E}\left(\widetilde{\tau}_{0, N}\right)\right) \sim(\log N)^{-2} \rightarrow 0$ as $N \uparrow \infty$. (iii) follows from this last statement. 


\section{References}

[1] Blom, G. Mean transition times for the Ehrenfest urn model. Adv. in Appl. Probab. 21, no. 2, 479-480, (1989).

[2] Claussen, J. C. Drift reversal in asymmetric coevolutionary conflicts: influence of microscopic processes and population size. European Physical Journal B 60, 391-399, (2007).

[3] Collet, P.; Martinez, S.; Méléard, S.; San Martin, J. Quasi-stationary distributions for structured birth and death processes with mutations. arXiv:0904.3468 (April 2009).

[4] Dette, H.; Fill, J. A.; Pitman, J.; Studden, W. J. Wall and Siegmund duality relations for birth and death chains with reflecting barrier. Dedicated to Murray Rosenblatt. J. Theoret. Probab. 10, no. 2, 349-374, (1997).

[5] Dynkin, E. B. Markov processes. Vols. I, II. Translated with the authorization and assistance of the author by J. Fabius, V. Greenberg, A. Maitra, G. Majone. Die Grundlehren der Mathematischen Wissenschaften, Bände 121, 122 Academic Press Inc., Publishers, New York; Springer-Verlag, Berlin-Göttingen-Heidelberg 1965.

[6] Ehrenfest P. \& T. Uber zwei bekannte Eingewände gegen das Boltzmannsche H-Theorem, Zeitschrift für Physik 8, 311-314, (1907).

[7] Feller, W. An introduction to probability theory and its applications, Vol. 1. John Wiley and Sons, Third Edition, New York, 1968.

[8] Fill, J. A. The passage time distribution for a birth-and-death chain: Strong stationary duality gives a first stochastic proof. arxiv.org/abs/0707.4042. Journal of Theoretical Probability (2009), to appear.

[9] Feinsilver, P.; Kocik, J. Krawtchouk polynomials and Krawtchouk matrices. Recent advances in applied probability, 115-141, Springer, New York, (2005).

[10] Flajolet, P.; Huillet, T. Analytic Combinatorics of the Mabinogion Urn. Fifth Colloquium on Mathematics and Computer Science: Algorithms, Trees, Combinatorics and Probabilities, U. Rösler (editor), Discrete Mathematics and Theoretical Computer Science Proceedings, vol. AI, p. 549572, (2008).

[11] Huillet, T. On Wright-Fisher diffusion and its relatives. J. Stat. Mech., Th. and Exp. P11006, vol. 11, (2007).

[12] Kac, M. Random walk and the theory of Brownian motion. Amer. Math. Monthly 54, 369-391, (1947).

[13] Kac, M. Probability and related topics in physical sciences. With special lectures by G. E. Uhlenbeck, A. R. Hibbs, and B. van der Pol. Lectures in Applied Mathematics. Proceedings of the Summer Seminar, Boulder, Colo., 1957, Vol. I Interscience Publishers, London-New York 1959 xiii+266 pp.

[14] Karlin, S.; McGregor, J. Ehrenfest urn models. J. Appl. Probability 2, 352-376, (1965).

[15] Keilson, J. Markov chain models - rarity and exponentiality. Applied Mathematical Sciences, 28. Springer-Verlag, New York-Berlin, 1979.

[16] Pollett, P.K. Quasi-stationary distributions; a bibliography. available at: http://www.maths.uq.edu.au/ ${ }^{\sim}$ pkp/papers/qsds/qsds.pdf, (regularly updated).

[17] Scalas, E., Martin,E.; Germano, G. Ehrenfest urn revisited: Playing the game on a realistic fluid model. Phys. Rev. E 76, 011104, (2007).

[18] Yaglom, A. M. Certain limit theorems of the theory of branching random processes. (Russian) /Doklady Akad. Nauk SSSR (N.S.)/ 56, 795-798, (1947). 